\title{
Two-Way Mutual-Structure-Based Public Opinion Communication System: An Analysis with Simulation
}

\author{
Weidong HUANG, Yang CUI, Xiang XIAO
}

\begin{abstract}
Mastering the evolutionary trend of an event paradox is significant for regulating event development. The powerful social synergy from aggregating public opinion can shape the development process and direction of an event and place tremendous pressure on society managers. Understanding the evolution of public opinion can help relevant departments better supervise and manage public opinion. This paper analyzes the generation path and propagation mode of public opinion through quantitative analysis and an interdisciplinary quotation of infectious disease models to establish a public opinion model based on a bidirectional mutual structure model of the paradoxgenerating path. The multi-agent simulation platform Netlogo is used to simulate a constructed public opinion evolution model, and the law of public opinion evolution under the resonance path is analyzed. Taking Stephen Hawking's death in 2018 as a sample case, simulation results are verified through data collection and collation; additionally, the law of the evolution of public opinion in the two-way mutual structure model is further summarized. This model can be compared with the evolution law of public opinion events. Such evolution can be roughly divided into three stages (diffusion, peak, and regression), which comprehensively explain the evolution of Weibo. The characteristics and behavioral mechanisms of each behavioral subject reflect the true event evolution. The model also provides a method for accurately judging event classification attributes and calculation formulas. This model can be applied in various public opinion supervision departments and provide a reference for social impacts following public opinion development.
\end{abstract}

Keywords: infectious disease model; Netlogo simulation; public opinion; public opinion evolution; two-way mutual structure

\section{INTRODUCTION}

Research regarding the evolution of public opinion has been mainly based on the guidance and social influence of public opinion. Given the proliferation of network information and the growing popularity of Internet applications, the openness, anonymity, freedom, and interactivity of the Internet have inspired hope that the network will express its own demands for social life. The Internet has thus become a prominent indicator of social public opinion. However, although the network can comprehensively reflect public opinion, it also spurs more sensationalism than traditional media. In addition, the Internet is relatively weak in terms of moral and administrative constraints and social order, which leads network-related public opinion to provide inconsistent information; such emotional and irrational details become reflected in real life and can lead to social grievances that adversely affect everyday life. Therefore, it is important to examine how to properly guide public opinion events and control public opinion to minimize negative effects on the event. Understanding the characteristics and law of development of public opinion - and assessing its dynamic evolution - can help relevant departments better monitor and manage such opinion, hence the focus of this study.

The concept of social dynamics [1] leverages multidisciplinary knowledge to study humans' social systems, explore individual differences in research problems, identify universal laws inherent in the system, and analyze microscopic phenomena emerging from macroscopic phenomena via simulation. When using social dynamics to explore microscopic features of complex problems, individual differences must be considered [2]. From the perspective of social dynamics, this article models a bidirectional mutual structure model of public opinion communication, analyzes the characteristics of subjects and objects in the model, formulates corresponding rules for subject-object interaction, and effectively quantifies the evolution process of public opinion. Subject interaction behavior is central to analyzing the macroscopic law of public opinion evolution [3]. It is also necessary to apply the classic model of public opinion communication (i.e., the infectious disease model). In this paper, a multi-agent simulation modeling method is proposed as an improvement upon the two-way mutual structure model.

\section{CONSTRUCTION OF TWO-WAY MUTUAL STRUCTURE MODEL}

\subsection{Traditional Infectious Disease Model}

Kermack and McKendrick developed the infectious disease model in 1927 to study the epidemic of the Black Death in London; the propagation process is shown in Fig. 1.

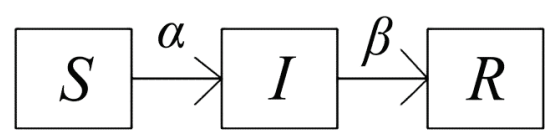

Figure 1 Schematic diagram of propagation process of infectious disease model

In this model, $S$ indicates a susceptible person, $I$ indicates an infected person, and $R$ indicates an immune person. In the epidemic infection process, the probability of $S$ being infected by $I$ is $\alpha$, which reflects the intensity of infection [4]. $I$ then becomes $R$, indicating that $I$ is cured, and its probability is $\beta$, which is also certain. Once the disease is cured, the person will produce antibodies and thus not be infected again; this is a unique attribute of $R$.

Later, because the process of infectious disease transmission is similar to that of information dissemination, the SIR model was applied to information dissemination research. In this use of the SIR model, all nodes are initially in an uninformed state $(S)$; that is, all individuals have no informational knowledge. Then some nodes receive this information and enter the diffusion state (I), attempting to spread the information to neighboring nodes in an uninformed state. Upon infecting a node (i.e., 
spreading information to an uninformed person), the diffuser node becomes immune $(R)$ and no longer participates in information dissemination.

\subsection{Description of Public Opinion Evolution Model Based on Two-way Mutual Structure Model}

According to the traditional infectious disease model, this paper applies the SIR-based infectious disease model to public opinion evolution. The proposed model of public opinion evolution is established based on the infectious disease model and the generated communication mode of public opinion (i.e., the two-way mutual structure model) as shown in Fig. 2.

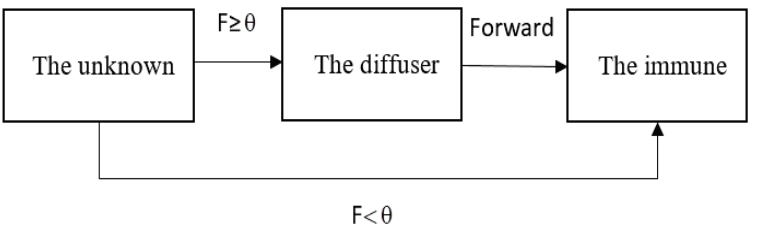

Figure 2 Public opinion evolution model

The proposed model operates as follows:

(1) In the process of public opinion evolution, a person who does not know public opinion information is the uninformed person $S$; the person who already knows public opinion information and can forward it is the proliferator, I; the individual who knows public opinion information but will not participate in dissemination is artificially immune, $R$.
(2) The rule of public opinion diffusion is that the user who already knows public opinion information (i.e., the proliferator) transmits the message to an uninformed person. Under certain conditions, the uninformed person becomes a proliferator; otherwise, immunity is altered. The broadcaster becomes immune after forwarding the sensational message, at which point the public opinion information is no longer disseminated.

(3) The spread of public opinion can be classified as either resonance or overflow. Traditional media reports elicit network public opinion and transmit relevant information through the network; after traditional media reports, they return to the network to continue to discuss two situations

(4) The beginning of the evolution of public opinion involves the emergence of information and proliferation of a public opinion event. The evolution ends when the public opinion information is no longer being diffused in the system space (or there are very few proliferators).

\subsection{Two-way Mutual Structure Model and Evolutionary Modeling of Public Opinion Generation}

According to the above-mentioned public opinion propagation model and analysis of the public opinion subject, object, and interaction path, this article constructs a two-way interaction model of public opinion generation (Fig. 3).

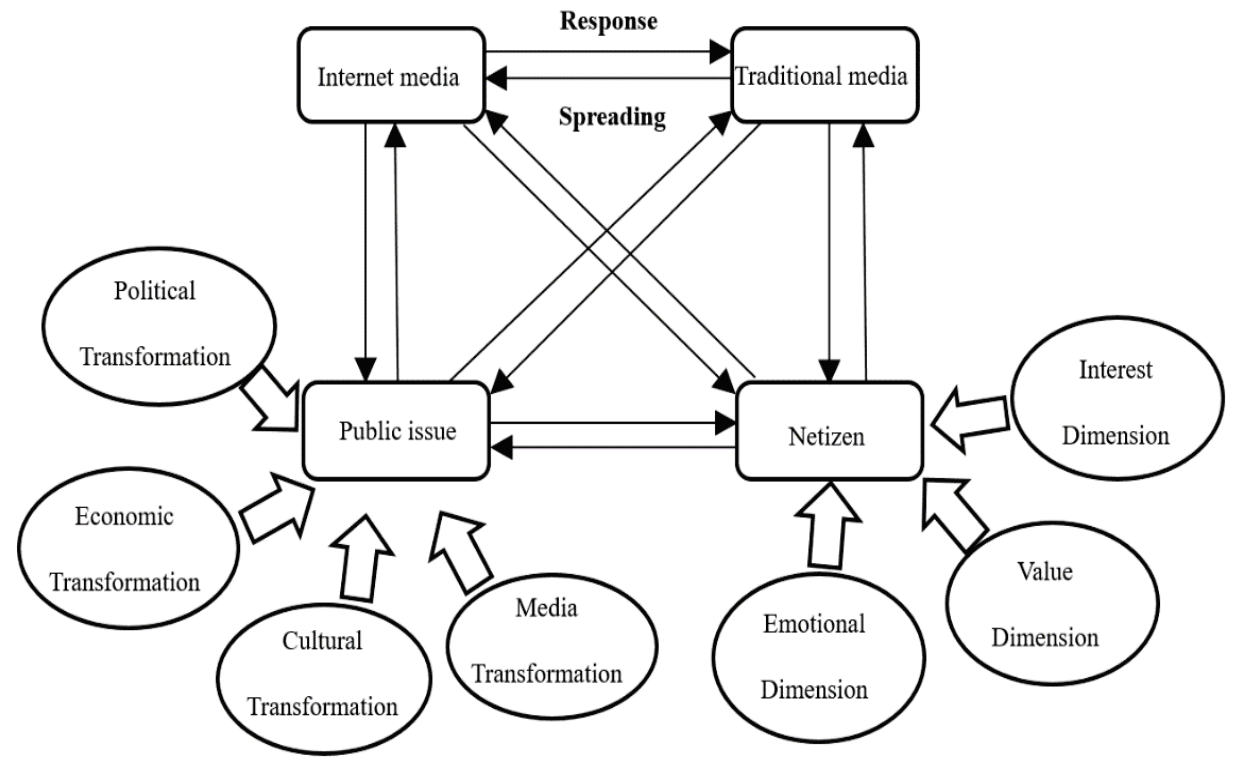

Figure 3 Bidirectional mutual structure pattern diagram generated by public opinion

\subsection{Description and Conditional Hypothesis of Public Opinion Evolution Model Based on Bidirectional Mutual Structure}

As noted earlier, this article refers to the SIR infectious disease model in examining public opinion evolution; the proposed model of public opinion evolution is rooted in the infectious disease model and the two-way mutual structure model. Given the principles outlined in Sec. 2.2, the proposed public opinion simulation model assumes the following regarding the state and attributes of each subject and object in the model. First, as original model data, the number of subjects and initial state of a given public opinion event are known. Second, the type of information of each subject is not considered in this study, and public opinion information is assumed; influential factors of ordinary netizens [5], online media, and traditional media are identical, thus eliciting the same attention. Third, in this study, news spread via online media in the process of public opinion evolution is assumed to be seen by most 
Internet users, followed by traditional media [7]. The average netizen has the smallest spread. Regarding the interaction rules between netizens and traditional media, traditional media is assumed to influence uninformed and immune individuals in the system.

\subsection{Subject-object Interaction Rules in Public Opinion Evolution}

The proposed model includes the following variables. For our purposes, influence and specialty respectively represent the influence and authority of online media; conformity refers to netizens' secularity; and significance and reliability represent the importance and credibility of public opinion information, respectively [9]. 'au' denotes authority and 'soio' denotes 'speed of information opening, which respectively represent the credibility of traditional media and the speed of information disclosure. co denotes conformity, indicating the belongingness of netizens. $S$ denotes significance, and $r$ denotes reliability. $i$ denotes influence, and $s p$ represents specialty. $p, q$, and $o$ respectively denote the number of ordinary netizens, the number of online media outlets, and the number of traditional media outlets.

\subsubsection{Parameter Setting of Main Object Attributes}

According to the above description of subject-object attributes in the evolution of public opinion, the parameters in this study are set as listed in Tab. 1.

Table 1 Subject and object attribute parameters of public opinion evolution

\begin{tabular}{|c|c|c|c|c|c|}
\hline $\begin{array}{l}\text { Attribute } \\
\text { parameter }\end{array}$ & Parameter definition & $\begin{array}{c}\text { Initial } \\
\text { value }\end{array}$ & $\begin{array}{l}\text { Attribute } \\
\text { parameter }\end{array}$ & Parameter definition & $\begin{array}{l}\text { Initial } \\
\text { value }\end{array}$ \\
\hline$p$ & Number of ordinary netizens & 500 & $q$ & Number of online media outlets & 5 \\
\hline$O$ & Number of traditional media outlets & 2 & $a u$ & Credibility of traditional media & 0.8 \\
\hline$s$ & Significance of public opinion information & 0.8 & soio & Traditional media information disclosure speed & 0.8 \\
\hline$r$ & Credibility of public opinion information & 0.8 & co & Generality of ordinary netizens & 0.7 \\
\hline$i$ & Influence of online media & 0.6 & $s p$ & Authority of online media & 0.6 \\
\hline$a_{1}$ & $\begin{array}{l}\text { How the secularity of netizens in netizen interactions } \\
\text { affects the weight of their status }\end{array}$ & 0.33 & $a_{2}$ & $\begin{array}{l}\text { How the significance of public opinion information in } \\
\text { netizen interactions affects the weight of their status }\end{array}$ & 0.33 \\
\hline$a_{3}$ & $\begin{array}{l}\text { How the credibility of public opinion information in } \\
\text { netizen interactions affects the weight of their status }\end{array}$ & 0.33 & $b_{1}$ & $\begin{array}{l}\text { How the influence of online media in netizens' } \\
\text { interactions with online media affects the weight of } \\
\text { netizens' status }\end{array}$ & 0.2 \\
\hline$b_{2}$ & $\begin{array}{l}\text { How the authority of online media in netizens' } \\
\text { interactions with online media affects the weight of } \\
\text { netizens'status }\end{array}$ & 0.2 & $b_{3}$ & $\begin{array}{l}\text { When netizens interact with online media, netizens' } \\
\text { belongingness affects the weight of netizens' status }\end{array}$ & 0.2 \\
\hline$b_{4}$ & $\begin{array}{l}\text { How the significance of public opinion information of } \\
\text { online media in netizens' interactions with online } \\
\text { media affects the weight of netizens' status }\end{array}$ & 0.2 & $b_{5}$ & $\begin{array}{l}\text { The credibility of public opinion information when } \\
\text { netizens interact with online media affects the weight } \\
\text { of netizens' status }\end{array}$ & 0.2 \\
\hline$c_{1}$ & $\begin{array}{l}\text { How the credibility of traditional media affects } \\
\text { netizens' status }\end{array}$ & 0.2 & $c_{2}$ & $\begin{array}{l}\text { When netizens interact with traditional media, the } \\
\text { speed of information disclosure of traditional media } \\
\text { affects the weight of netizens' status }\end{array}$ & 0.2 \\
\hline$c_{3}$ & $\begin{array}{l}\text { How the secular nature of netizens' interactions with } \\
\text { traditional media affects the weight of netizens' status }\end{array}$ & 0.2 & $c_{4}$ & $\begin{array}{l}\text { The significance of public opinion information when } \\
\text { netizens interact with traditional media affects the } \\
\text { weight of netizens' status }\end{array}$ & 0.2 \\
\hline$c_{5}$ & $\begin{array}{l}\text { The extent to which the credibility of public opinion } \\
\text { information when netizens interact with traditional } \\
\text { media affects the weight of netizens' status }\end{array}$ & 0.2 & & & \\
\hline
\end{tabular}

\subsubsection{Netizen Interaction Rules}

If ordinary netizen $n$ is uninformed and netizen $m$ is a diffuser, then the influence function of a netizen on $n$ at time $t$ is

$$
\begin{aligned}
& f=(c o) \cdot a_{1}+s \cdot a_{2}+r \cdot a_{3} \\
& a_{1}, a_{2}, a_{3} \in[0,1] \\
& a_{1}+a_{2}+a_{3}=1
\end{aligned}
$$

where $f$ represents the influence function of a netizen on $n$ at time $t$. Eq. (2) indicates the degree of the netizen's belongingness. Eq. (3) indicates the weight of the significance and credibility of public opinion. Here, $\theta$ is a constant whose value range is $[0,1]$. If $f<\theta, n$ is converted from an uninformed person to an immune person; if $\theta \leq f \leq$ $1, n$ is converted from an uninformed person to a diffuser. If $n$ is a diffuser or immune, then $m$ has no effect on $n$ 's state. After netizen $m$ disseminates the sensational message, he or she changes from a diffuser to an immunizer.

\subsubsection{Interaction Rules between Netizens and Online Media}

If a netizen is uninformed at time $t$, the influence function of online media on that netizen is as follows:

$$
\begin{aligned}
& h=i \cdot b_{1}+(s p) \cdot b_{2}+(c o) \cdot b_{3}+s \cdot b_{4}+r \cdot b_{5} \\
& b_{1}, b_{2}, b_{3}, b_{4}, b_{5} \in[0,1] \\
& b_{1}+b_{2}+b_{3}+b_{4}+b_{5}=1
\end{aligned}
$$

where $h$ represents the influence function of online media. Eq. (5) indicates the weight of the influence and the authority of online media. Eq. (6) indicates the weight of the significance of public opinion information and the credibility of the netizen's belongingness. If $h<\theta, n$ is converted from an uninformed to an immune person; if $\theta \leq$ $h \leq 1, n$ is converted from an uninformed person to a diffuser. Again, $\theta$ is a constant whose value range is $[0,1]$. Its value is situation-dependent; if $n$ is a diffuser or immune, then online media has no effect on the netizen's status. 
The state of online media outlet $k$ (whether concerning a diffuser or immunizer) depends on clique, denoting the number of distributors in the system space at time $t$. Given the total number of subjects $(p+q+o)$, the state function of online media outlet $k$ can be expressed as the significance and reliability of public opinion information such that

$$
\begin{aligned}
& H=\frac{(\text { clique }) \cdot b_{6}}{p+q+o}+s \cdot b_{7}+r \cdot b_{8} \\
& b_{6}+b_{7}+b_{8}=1 \\
& b_{6}, b_{7}, b_{8} \in[0,1]
\end{aligned}
$$

where $H$ denotes the state function of online media outlet $k ; b_{6}$ denotes the weight of the broadcaster to the state of online media outlet $k$; and $b_{7}$ and $b_{8}$ respectively denote the weight of public opinion information and credibility to the state of online media outlet $k$. If $H<\theta_{1}$, online media $k$ is immune; if $\theta_{1} \leq H \leq 1$, online media $k$ is a diffuser. $\theta_{1}$ is a constant belonging to the interval of $[0,1]$, and its value is situation-dependent. After online media has disseminated sensational news, then the network changes from a diffuser to an immunizer.

\subsubsection{Interaction Rules between Netizens and Traditional Media}

If the state of a netizen at time $t$ is either uninformed or immune, the influence of traditional media on netizens is expressed as

$$
\begin{aligned}
& g=(\text { au }) \cdot c_{1}+(\text { soio }) \cdot c_{2}+(\text { co }) \cdot c_{3}+s \cdot c_{4}+r \cdot c_{5} \\
& c_{1}+c_{2}+c_{3}+c_{4}+c_{5}=1 \\
& c_{1}, c_{2}, c_{3}, c_{4}, c_{5} \in[0,1]
\end{aligned}
$$

where $g$ represents the influence of traditional media on netizens. Eqs. (11) and (12) respectively indicate the credibility of traditional media; and the speed of information disclosure, belongingness of netizens, significance of public opinion, and weight of credibility. If $g<\theta, n$ is converted from an uninformed to an immunized person; if $\theta \leq g \leq 1, n$ is converted from an uninformed person to a diffuser. Here, $\theta$ is a constant with the range of $[0,1]$, and its value is situation-dependent. If $n$ is immune, then traditional media has no effect on his or her state.

We define the state of traditional media as a time function of the speed at which public information is disclosed:

$$
\begin{aligned}
& G=(\text { soio }) \cdot c_{6} \\
& c_{6} \in[0,100]
\end{aligned}
$$

where $G$ represents the state of traditional media, and $c_{6}$ is the time parameter whose value is random depending on the situation. Here, $\theta_{2}$ is a constant belonging to the interval $[0,100]$. If $G<\theta_{2}$, the conventional media $T$ is an immunizer; if $\theta_{2} \leq G \leq 1$, the conventional media $T$ is a diffuser. After traditional media has disseminated sensational news, its state changes from a diffuser to an immunizer.

\section{SIMULATION AND EMPIRICAL STUDY OF PUBLIC OPINION EVOLUTION BASED ON TWO-WAY MUTUAL STRUCTURE MODEL \\ 3.1 Fundamentals of Public Opinion Evolution Modeling}

The basic idea of public opinion evolution modeling is to analyze the attributes and interaction rules of each subject and object system according to the two-way mutual structure generated by public opinion, after which a multiagent public opinion simulation model can be established. For the purposes of this paper, the model was simulated on via Netlogo and analyzed. Simulation results demonstrate the evolution of public opinion in the two paths of overflow and resonance [8]. We then examine the evolution of actual public opinion events based on a real-life sample case. Finally, we verify the simulation results of the model based on our analysis to further refine the model. The multisubject public opinion simulation model is depicted in Fig. 4

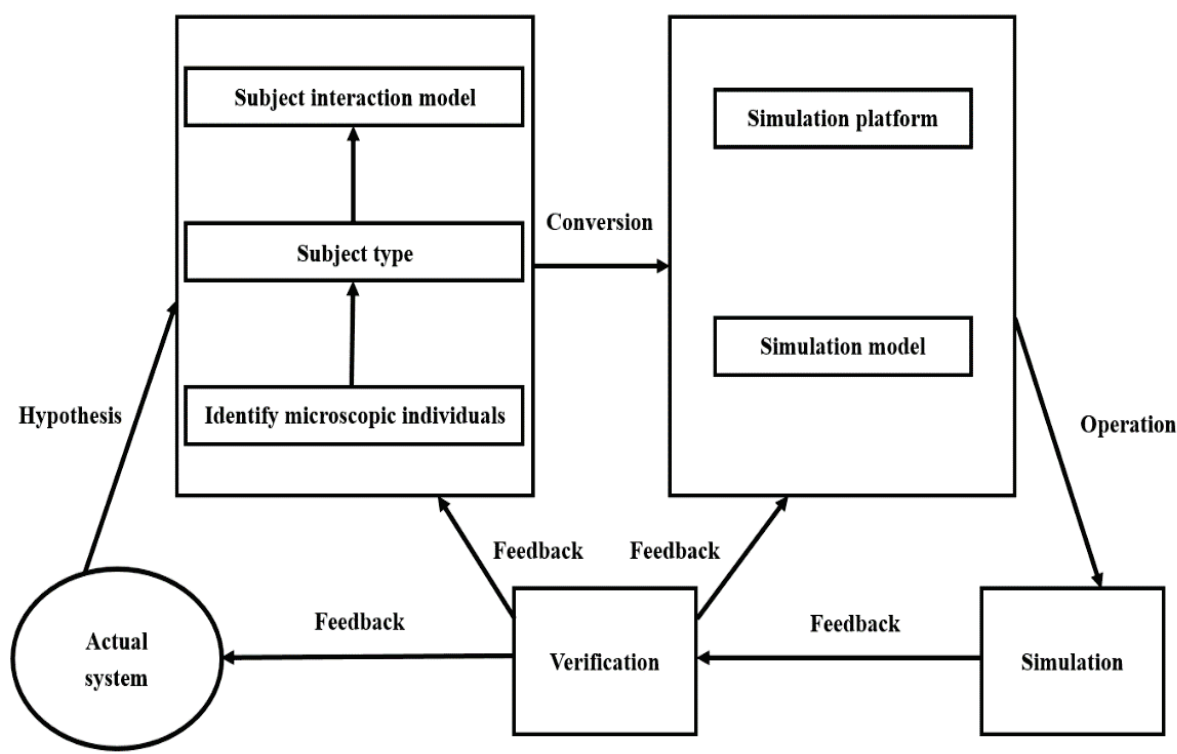

Figure 4 Basics of public opinion evolution modeling and simulation 


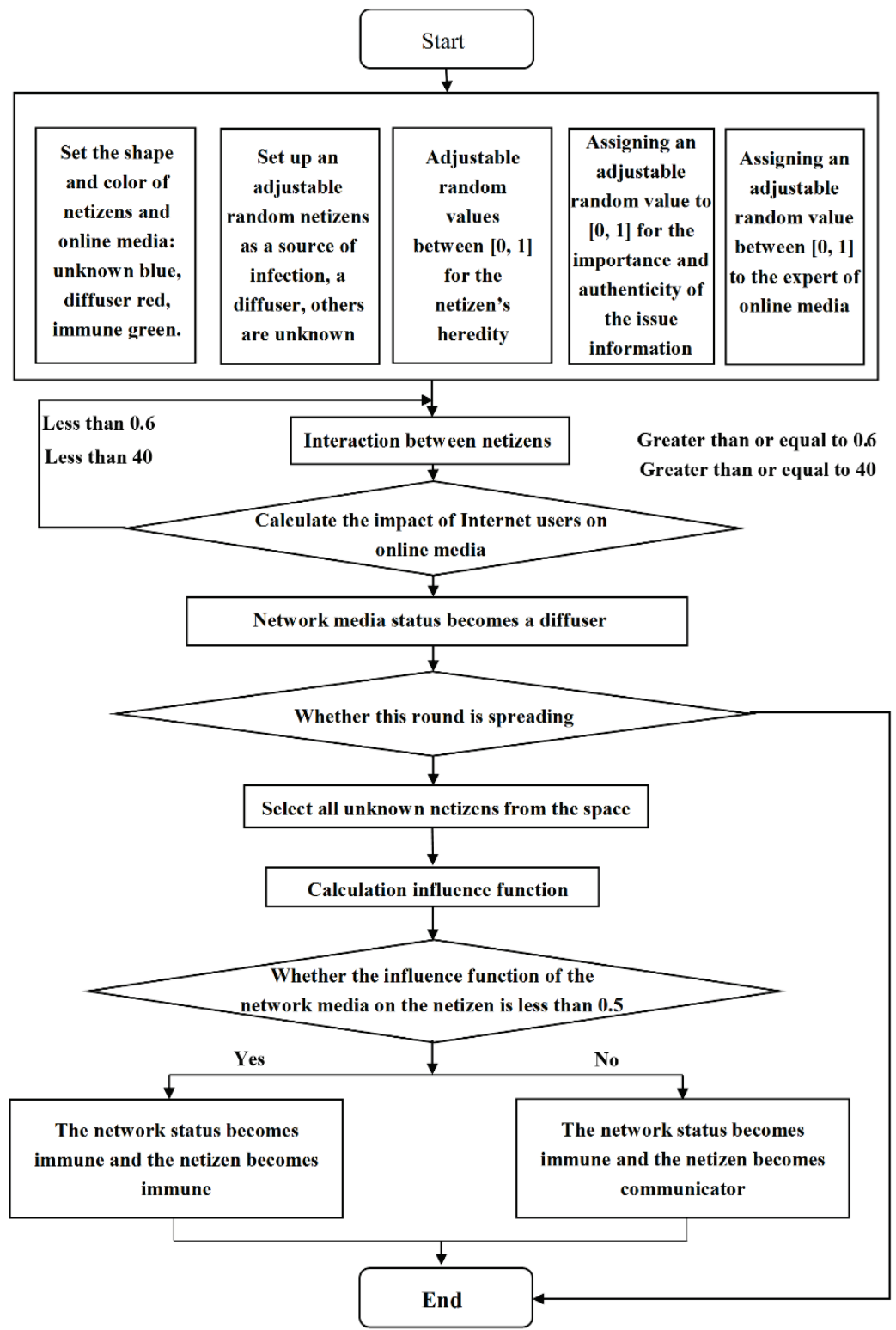

Figure 5 Public opinion information interaction flow chart

\subsection{Simulation Design Process}

Because interactions proliferate between ordinary netizens, no judgment statement is included; see Fig. 5, which illustrates the flow of public opinion information interaction between ordinary netizens and online media [6] and between ordinary netizens and traditional media. The two key points of 0.6 and 40 respectively determine the impact of ordinary netizens and networks/traditional media in the simulation process.

\section{SIMULATION RESULTS AND EXPERIMENTAL ILLUSTRATION ANALYSIS}

\subsection{Analysis of Evolution Process of Public Opinion in the Resonance Path}

According to the path generated by the abovementioned public opinion, resonance indicates that traditional media reports lead to network public opinion. Given the increasing influence of network public opinion on society, social public opinion and network public opinion in the resonance path can have similar effect.
Therefore, the evolution process of the public opinion event examined in this study, in terms of the resonance path, is as follows: the initial stage takes traditional media as the source of dissemination, and then netizens and online media participate in public opinion diffusion simultaneously. Simulation results are presented in Fig. 6.

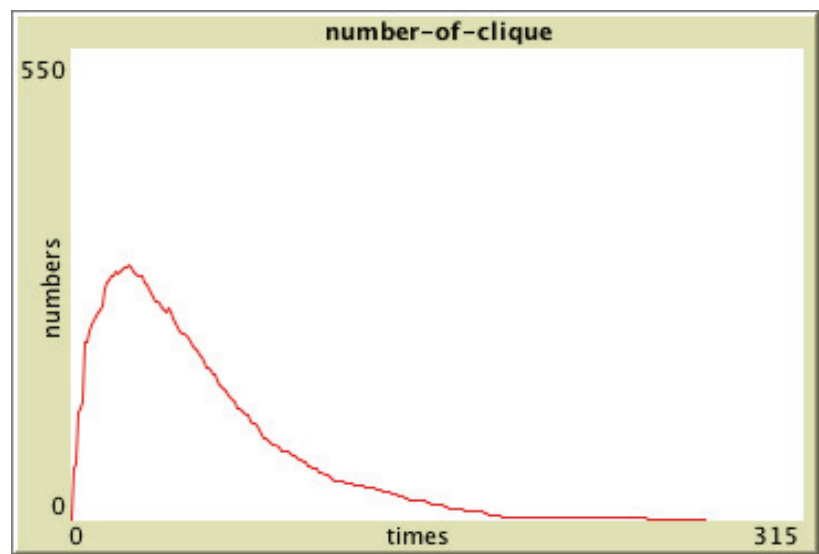

Figure 6 Simulation analysis of public opinion evolution under resonance path 
Under the resonance path, due to the broad influence of traditional media, the number of diffusers in the system exhibits rapid growth followed by a peak and gradual decline. The resulting evolution of public sentiment proceeds similarly. Throughout this process, traditional media serves as the primary information source, while netizens and online media are the recipients and diffusers of information.

\subsection{Analysis of Public Opinion Evolution of Physicist Stephen Hawking's Death}

Based on the above results, we selected an appropriate sample case to verify the rationality and accuracy of the simulation. On March 14, 2018, according to BBC, Guardian, and many other foreign media reports, the famous physicist Stephen Hawking died at his home in Cambridge, England at age 76. After confirmation, many media outlets in China reported the news and caused a sensational frenzy. Relevant keywords first appeared on Weibo.

The authors of this study used "Hawking's death" as a keyword along with statistics from the heat index of "Physicist Hawking's death" on the Baidu index platform and posting volume on Baidu Post Bar (topic-only posts) obtained between March 13 and April 13, 2018; see Figs. 7 and 8 .

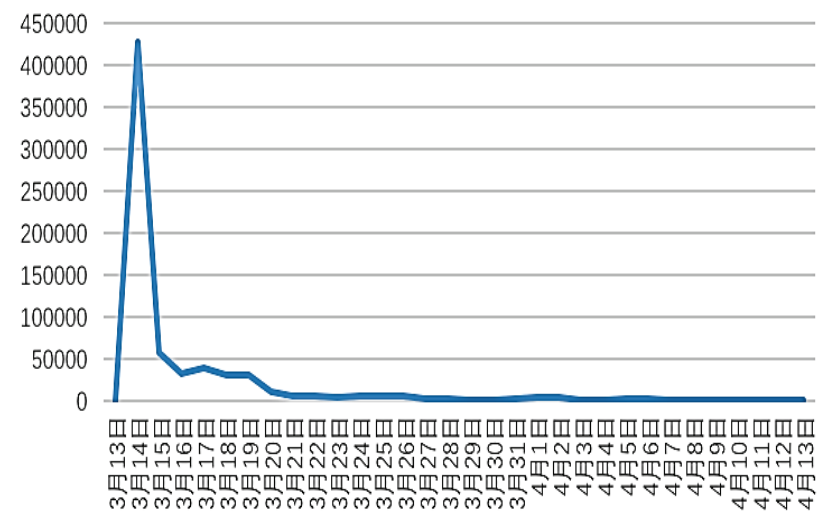

Figure 7 Physicist Hawking's death: Baidu index trend chart

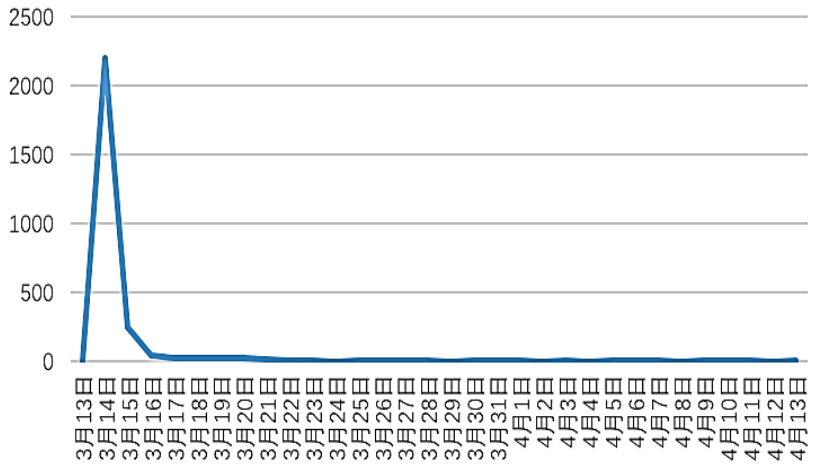

Figure 8 Physicist Hawking's death: Baidu Post Bar Posting Trend Chart

The Baidu index of the event and users' posting trends on Baidu Post Bar were nearly identical and each underwent three stages of rapid growth, peak, and gradual decline, reflecting public opinion under the resonance path. According to our simulation analysis of the model under the resonance path, the evolution of public opinion also underwent these three stages; therefore, the proposed model can better reflect the evolution process of public opinion under the resonance path. In this case, traditional media served as the main source of information (i.e., the source of sensationalism), while netizens and online media were both recipients and proliferators of public opinion information.

\section{RELATED DISCUSSION AND ANALYSIS}

Findings from this study reveal that the emergence and intensification of social contradictions are inevitable in social transformation, and public opinion events generated under the resonance path fall under the development and impact of media. Internet users take the network as an open platform to participate in events, thus promoting the evolution of information disclosure. Traditional media should factually investigate public opinion incidents and guide such opinion to prevent dissemination of inaccurate or potentially harmful information. Through the resonance path for positive interaction, traditional media and online media can promote event development and disclosure of government information.

In the next phase of this study, subject and object attributes will be considered more comprehensively. The influence of public opinion information on real-life actors may be found to be different. The research process will also be refined, and model and simulation results will be more precise.

\section{CONCLUSION}

Analysis of the model simulation results and sample case reveals the following:

(1) According to the two-way mutual structure model generated by public opinion (based on the infectious disease model), the multi-agent simulation platform Netlogo can be used to simulate the evolution process of public opinion in the resonance path, and the law of the evolution of public opinion in this path can be analyzed. The mechanism of action of each behavioral subject is as follows: 1) the generation of public opinion events under the resonance path is faster and can evoke a strong social paradox over an extended period of time; 2) throughout public opinion evolution, netizens, online media outlets, and traditional media outlets serve as sources of public issues, information recipients, and proliferators;

(2) The sample case verified the model simulation results, substantiating the adaptability of the model and accuracy of the simulation;

(3) Regarding the evolution law of public opinion, 1) under the resonance path, evolution generally proceeds through three stages: rapid growth, peak, and gradual decline. Event evolution can be examined comprehensively, and the evolution of public opinion can be divided into diffusion, peak, and regression.

In the development of public opinion, netizens, online media, and traditional media serve numerous roles. Traditional media reports on public events may inspire netizens to explore an event further. Netizens' opinions can also be disseminated through various online channels 
based on public issues initiated by the network. The rapid development of social transformation and network information has rendered information dissemination unprecedentedly active, affording citizens with reasonable discourse power and presenting obstacles to the stable management of society. Our findings quantify the interaction between subjects and objects in terms of network public opinion evolution, and the simulation model reflects the evolution of a chosen public opinion event. Results regarding the process of public opinion evolution can help relevant departments understand the formation and evolution of public opinion and then formulate a reasonable public opinion control strategy, which carries important theoretical and practical implications.

\section{Acknowledgements}

Work described in this paper was funded by the National Natural Science Foundation of China under Grant No. 71671093. The authors would like to thank other researchers at Nanjing University of Posts and Telecommunications.

This work was also supported by the Jiangsu Provincial Department of Education, the Key Research Base of Philosophy and Social Sciences; and by the Jiangsu Province University Philosophy and Social Sciences Outstanding Innovation Team Construction Project.

\section{REFERENCES}

[1] Weidlich, W. (2003). Sociodynamics - a systematic approach to mathematical modelling in the social sciences. Chaos, Solitons \& Fractals, 18(3), 431-437. https://doi.org/10.1016/S0960-0779(02)00666-5

[2] Cioffi-Revilla, C. (2014). Introduction to computational social science. New York: Springer https://doi.org/10.1007/978-1-4471-5661-1

[3] Marchi, S. \& Page. S. E. (2014). Agent-based modeling. Annual Review of Political Science, 17(1), 1-20. https://doi.org/10.1146/annurev-polisci-080812-191558

[4] Matsubara, Y., Sakurai, Y., Prakash, B. A., Li, L., \& Faloutsos, C. (2012, August). Rise and fall patterns of information difusion: model and implications. In Proceedings of the $18^{\text {th }}$ ACM SIGKDD international conference on Knowledge discovery and data mining, ACM, 6-14. https://doi.org/10.1145/2339530.2339537

[5] Gilbert, N. (2008). Agent-based models. New York: SAGE Publications. https://doi.org/10.4135/9781412983259

[6] Tiance, D. \& Ying, C. (2006). On the interactive interaction between traditional media and online media. Inaugural Meeting of China Communication Society and the Ninth National Communication Symposium. 134-138.

[7] Jingtai, T. (2015). The management of integrated issues in the guidance of public opinions in the context of new media.China Emergency Management, 2015(07), 37-41.

[8] Zhu, W., Wan, M., Zhou, Y., \& Pan, W. (2018). Fuzzy computation of teaching performance based on data envelopment analysis method. Cognitive Systems Research, 52, 351-358. https://doi.org/10.1016/j.cogsys.2018.07.018

[9] Pan, W. T. (2017). A Newer Equal Part Linear Regression Model: A Case Study of the Influence of Educational Input on Gross National Income. Eurasia Journal of Mathematics, Science and Technology Education, 13(8), 5765-5773. https://doi.org/10.12973/eurasia.2017.01026a

\section{Contact information:}

\section{Weidong HUANG}

Nanjing University of Posts and Telecommunications,

No. 66, XinmaoFanma Road, Central Gate Street, Gulou District, Nanjing, Jiangsu, China, 210000

E-mail: huangwd@njupt.edu.cn

Yang CUI

Nanjing University of Posts and Telecommunications,

No. 66, XinmaoFanma Road, Central Gate Street, Gulou District, Nanjing, Jiangsu, China, 210000

E-mail: cuiyang9515@163.com

\section{Xiang XIAO}

Nanjing University of Posts and Telecommunications,

No. 66, XinmaoFanma Road, Central Gate Street, Gulou District, Nanjing, Jiangsu, China, 210000

E-mail:1941733156@qq.com 